\title{
Table for Third-Degree Spline Interpolation With Equally Spaced Arguments*
}

\author{
By T. N. E. Greville
}

\begin{abstract}
A table is given to facilitate the calculation of the parameters of the interpolating third-degree natural spline function for $n$ given data points $(n>2)$ with equally spaced abscissas. The use of the table is described and the correctness of the algorithm is demonstrated.
\end{abstract}

1. Introduction. Given a set of $n$ real numbers $x_{1}<x_{2}<\cdots<x_{n}$ called "knots," a spline function of degree $m$ having the knots $x_{j}$ is defined to be a function $S(x)$ satisfying the following two conditions:

(1) In each interval $\left(x_{j}, x_{j+1}\right)\left(j=0,1, \cdots, n ; x_{0}=-\infty, x_{n+1}=\infty\right), S(x)$ is given by some polynomial of degree $m$ (or less).

(2) The polynomial arcs which represent the function in successive intervals join smoothly in the sense that $S(x)$ and its derivatives of order $1,2, \cdots, m-1$ are continuous over $(-\infty, \infty)$.

A spline function of odd degree $2 k-1$ is called a "natural" spline function if it satisfies the further condition:

(3) In each of the two intervals $\left(-\infty, x_{1}\right)$ and $\left(x_{n}, \infty\right) S(x)$ is represented by a polynomial of degree $k-1$ or less (in general, not the same polynomial in the two intervals).

It is well known [1] that given any set of $n$ data points $\left(x_{j}, y_{j}\right)$ with distinct abscissas, and an integer $k \leqq n$, there is a unique natural spline function $s(x)$ of degree $2 k-1$, having its knots limited to the abscissas $x_{j}$, that also interpolates the given data points, in the sense that $s\left(x_{j}\right)=y_{j}(j=1,2, \cdots, n)$. Moreover, in the class of continuous functions $f(x)$ with continuous derivatives of order $1,2, \cdots$, $k$ on $(-\infty, \infty)$, this natural spline interpolating function is the "smoothest" interpolating function for the given data points, in the sense that the integral

$$
\int_{a}^{b}[f(x)]^{2} d x
$$

(for any $a, b$ such that $a \leqq x_{1}$ and $b \geqq x_{n}$ ) is smallest.

Third-degree spline functions (i.e., $k=2$ ) have been much more widely used than those of any other degree, and an algorithm is given in [1] for obtaining the third-degree interpolating natural spline function for any set of ( 2 or more) given data points with distinct abscissas. This algorithm involves the solution of an $(n-2) \times(n-2)$ tridiagonal system of linear equations.

Received May 26, 1969.

AMS Subject Classifications. Primary 6505, 6520; Secondary 4110, 4130.

Key Words and Phrases. Natural cubic spline interpolation, smoothest interpolating function, generating functions.

* Sponsored by the Mathematics Research Center, United States Army, Madison, Wisconsin, under contract No.: DA-31-124-ARO-D-462. 
If the abscissas of the data points are equally spaced, substantial simplification is possible, and the parameters of the third-degree interpolating natural spline function can be obtained explicitly, by the use of the table contained in this report, without the necessity of solving a system of equations.

2. Use of the Table. It is assumed that suitable changes of origin and scale have been made, if necessary, so that $x_{j}=j(j=1,2, \cdots, n)$. On this assumption $s(x)$ can be expressed [1] in the form

$$
s(x)=s(1)+(x-1) d+\sum_{j=1}^{n} c_{j}(x-j)_{+}{ }^{3},
$$

where the truncated power function $z_{+}{ }^{3}$ is given by

$$
\begin{aligned}
z_{+}{ }^{3} & =z^{3} & & (z \geqq 0) \\
& =0 & & (z<0) .
\end{aligned}
$$

The coefficients $d$ and $c_{j}$ are to be determined.

TABLE 1

\begin{tabular}{|c|c|c|}
\hline$j$ & $\alpha_{j}$ & $\beta_{j}$ \\
\hline 2 & 1 & 1 \\
\hline 3 & -6 & -4 \\
\hline 4 & 24 & 15 \\
\hline 5 & -90 & -56 \\
\hline 6 & 336 & 209 \\
\hline 7 & -1254 & -780 \\
\hline 8 & 4680 & 2911 \\
\hline 9 & -17466 & -10864 \\
\hline 10 & 65184 & 40545 \\
\hline 11 & -243270 & -151316 \\
\hline 12 & 907896 & 5) 64719 \\
\hline 13 & -3388314 & -2107560 \\
\hline 14 & 12645360 & 7865521 \\
\hline 15 & -47193126 & $-293 \quad 54524$ \\
\hline 16 & $1761 \quad 27144$ & 109552575 \\
\hline 17 & -657315450 & -408855776 \\
\hline 18 & 2453134656 & 1525870529 \\
\hline 19 & -9155223174 & -5694626340 \\
\hline 20 & 34167758040 & 21252634831 \\
\hline
\end{tabular}

Constants for Calculating Third-Degree Interpolating

Natural Spline Function for Equally Spaced Arguments

The table can be continued by means of the following relations (the first of which does not hold for $j=3$ ):

$$
\begin{aligned}
\alpha_{j+1} & =-4 \alpha_{j}-\alpha_{j-1} \\
\beta_{j+1} & =-4 \beta_{j}-\beta_{j-1} \\
\alpha_{j} & =\beta_{j}-2 \beta_{j-1}+\beta_{j-2}
\end{aligned}
$$

Table 1 gives the values of integer constants $\alpha_{j}$ and $\beta_{j}$ corresponding to each integer $j \geqq 2$. The coefficient $d$ is given by 


$$
d=\left[\alpha_{2}\left(y_{n}-y_{1}\right)+\alpha_{3}\left(y_{n-1}-y_{1}\right)+\cdots+\alpha_{n}\left(y_{2}-y_{1}\right)\right] / \beta_{n} .
$$

In order to avoid very rapid accumulation of rounding error (which would otherwise be a serious problem if $n$ is even moderately large), it is suggested that the division by $\beta_{n}$ be postponed. Thus $d$ would be retained in the form $N / \beta_{n}$, where $N$ is calculated exactly, using integer or fixed-point arithmetic.

The quantities $\beta_{n} c_{j}(j=1,2, \cdots, n)$ are then obtained recursively by the formulas

$$
\begin{aligned}
& \beta_{n} c_{1}=\beta_{n}\left(y_{2}-y_{1}\right)-N, \\
& \beta_{n} c_{j}=\beta_{n}\left(y_{j+1}-y_{1}\right)-j N-2^{3} \beta_{n} c_{j-1}-3^{3} \beta_{n} c_{j-2}-\cdots-j^{3} \beta_{n} c_{1} \\
& \quad(j=2,3, \cdots, n-1),
\end{aligned}
$$

again using exact calculation throughout. (The quantities $y_{j}-y_{1}$ must, of course, be actually multiplied by $\beta_{n}$.) Finally, $N$ and the quantities $\beta_{n} c_{j}$ are divided by $\beta_{n}$ to give the parameters $d$ and $c_{j}$ to the desired precision. It should be borne in mind that in the expression (2.1) the coefficients $c_{j}$ (especially those with smaller indices) will sometimes be multiplied by large numbers, and may be needed to many decimal places.

3. Derivations and Proofs. Taking $x=k+1$ in (2.1), transposing certain terms, and noting that $s(k)=y_{k}$ for $k=1,2, \cdots, n$ gives at once

$$
c_{k}=y_{k+1}-y_{1}-k d-2^{3} c_{k-1}-3^{3} c_{k-2}-\cdots-k^{3} c_{1},
$$

from which (2.4) follows immediately. Similarly, taking $x=2$ gives (2.3).

Let $\phi(x)$ denote the infinite series

$$
\phi(x)=1^{3}+2^{3} x+3^{3} x^{2}+\cdots,
$$

which converges in the interior of the unit circle. By actual multiplication

$$
(1-x)^{4} \phi(x)=1+4 x+x^{2},
$$

and therefore

$$
\phi(x)=\frac{1+4 x+x^{2}}{(1-x)^{4}} .
$$

Further, let

$$
\eta(x)=\sum_{j=2}^{\infty}[s(j)-s(1)] x^{j-2} .
$$

As $s(x)$ is a linear function for $x \geqq n$, this series also converges within the unit circle, as does the binomial expansion

$$
(1-x)^{-2}=1+2 x+3 x^{2}+\cdots .
$$

Finally, we denote by $C(x)$ the polynomial

$$
C(x)=c_{1}+c_{2} x+\cdots+c_{n} x^{n-1} .
$$


From (2.1), (3.1), (3.3), (3.4) and (3.5) we obtain the identity

$$
\eta(x)=d(1-x)^{-2}+\phi(x) C(x) .
$$

Now, let

$$
\psi(x)=\frac{1}{1+4 x+x^{2}} .
$$

Clearly its Maclaurin expansion

$$
\psi(x)=\sum_{j=0}^{\infty} b_{j} x^{j}=1-4 x+15 x^{2}-\cdots
$$

converges in a neighborhood of the origin. Multiplying (3.6) by $(1-x)^{2} \psi(x)$ gives

$$
(1-x)^{2} \psi(x) \eta(x)=d \psi(x)+(1-x)^{-2} C(x),
$$

where we have used (3.2) and (3.7). It is shown in [1] that the coefficients $c_{j}$ satisfy the two conditions

$$
\begin{aligned}
c_{1}+c_{2}+\cdots+c_{n} & =0, \\
c_{1}+2 c_{2}+\cdots+n c_{n} & =0 .
\end{aligned}
$$

Incidentally, (2.5) follows from (3.10).

Returning, however, to (3.9), we equate coefficients of $x^{n-2}$ on both sides of that equation, noting that the coefficient of $x^{n-2}$ in $(1-x)^{-2} C(x)$ is

$$
\begin{aligned}
(n-1) c_{1}+(n-2) c_{2} & +\cdots+2 c_{n-2}+c_{n-1} \\
& =n\left(c_{1}+c_{2}+\cdots+c_{n}\right)-\left(c_{1}+2 c_{2}+\cdots+n c_{n}\right)=0,
\end{aligned}
$$

by (3.10) and (3.11). Further, let

$$
(1-x)^{2} \psi(x)=\sum_{j=0}^{\infty} a_{j} x^{j},
$$

a series having the same region of convergence as that in (3.8). We obtain, therefore,

$$
a_{0}\left(y_{n}-y_{1}\right)+a_{1}\left(y_{n-1}-y_{1}\right)+\cdots+a_{n-2}\left(y_{2}-y_{1}\right)=d b_{n-2} .
$$

Finally, we redesignate the coefficients $a_{j}$ and $b_{j}$ as $\alpha_{j}$ and $\beta_{j}$, shifting the indices (for notational convenience in the use of Table 1) so that $\alpha_{j}=a_{j-2}$ and $\beta_{j}=b_{j-2}$. Making these substitutions in (3.13) at once gives (2.2). The recurrence relation for the quantities $\alpha_{j}$ follows from (3.7) and (3.12); that for the $\beta_{i}$ from (3.7) and (3.8). The relation $\alpha_{j}=\beta_{j}-2 \beta_{j-1}+\beta_{j-2}$ is an immediate consequence of (3.8) and (3.12).

4. Illustrative Example. The values of $j$ and $y_{j}$ in Table 2, due to K. A. Innanen [2], represent ten points on a segment of a theoretical rotation curve of the galactic system. Here $y_{j}$ is the circular velocity in the galactic plane in $\mathrm{km} / \mathrm{sec}$ at a distance of $j$ kiloparsecs from the galactic center. Substituting in (2.2) the values of $\alpha_{j}$ from Table 1 and those of $y_{j}-y_{1}$ from Table 2 gives

$$
\begin{aligned}
d & =[1(-24.0)-6(-22.5)+24(-23.0)-\cdots+65184(-23.0)] / 40545 \\
& =-1005780 / 40545=-67052 / 2703=-24.8065 .
\end{aligned}
$$


TABLE 2

Illustrative Data

\begin{tabular}{rcrrr}
\hline$j$ & $y_{j}$ & $y_{j}-y_{1}$ & \multicolumn{1}{c}{$2703 c_{j}$} & \multicolumn{1}{c}{$c_{j}$} \\
\hline 1 & 244.0 & 0.0 & 4883.0 & 1.8065 \\
2 & 221.0 & -23.0 & -2268.0 & -0.8391 \\
3 & 208.0 & -36.0 & -9849.0 & -3.6437 \\
4 & 208.0 & -36.0 & 7876.5 & 2.9140 \\
5 & 211.5 & -32.5 & -2736.0 & -1.0122 \\
6 & 216.0 & -28.0 & 3067.5 & 1.1349 \\
7 & 219.0 & -25.0 & -1425.0 & -0.5272 \\
8 & 221.0 & -23.0 & -70.5 & -0.0261 \\
9 & 221.5 & -22.5 & 1707.0 & 0.6315 \\
10 & 220.0 & -24.0 & -1185.5 & -0.4386 \\
\hline
\end{tabular}

Values of $2703 c_{j}$ are calculated exactly, using (2.3), (2.4), and (2.5). Finally, division by 2703 gives the values of $c_{j}$, shown in the last column of Table 2 to four decimal places. Thus, the third-degree interpolating natural spline function for these data is

$$
\begin{aligned}
244.0 & -24.8065(x-1)+1.8065(x-1)_{+}{ }^{3}-0.8391(x-2)_{+}{ }^{3} \\
& -3.6437(x-3)_{+}{ }^{3}+2.9140(x-4)_{+}{ }^{3}-1.0122(x-5)_{+}{ }^{3} \\
& +1.1349(x-6)_{+}{ }^{3}-0.5272(x-7)_{+}{ }^{3}-0.0261(x-8)_{+}{ }^{3} \\
& +0.6315(x-9)_{+}{ }^{3}-0.4386(x-10)_{+}{ }^{3} .
\end{aligned}
$$

Mathematics Research Center, U. S. Army

University of Wisconsin

Madison, Wisconsin 53706

1. T. N. E. Greville, "Spline functions, interpolation, and numerical quadrature," in Mathematical Methods for Digital Computers, Vol. 2, A. Ralston and H. S. Wilf (Editors), Wiley, New York, 1967, Chapter 8. MR $35 \# 2516$.

2. K. A. INNANEN, "An example of precise interpolation with a spline function," J. Computational Phys., v. 1, 1967, pp. 303-304. 